\title{
Synchrophasors Based Wide Area Protection and Phasor Estimation: A Review
}

\author{
Muhammad Qasim Khan \\ Musse Mohamud Ahmed \\ Department of Electrical \& Electronics \\ Faculty of Engineering \\ Universiti Malaysia Sarawak \\ Kota Samarahan, Malaysia
}

\author{
Ahmed M. A. Haidarab \\ Department of Electrical \& Electronics \\ ${ }^{a}$ Universiti Malaysia Sarawak \\ Samarahan, Sarawak, Malaysia \\ bUniversity of Southern Queensland \\ Toowoomba, Queensland, Australia
}

\author{
Norhuzaimin Julai \\ Mohammad Kamarul Hasan \\ Department of Electrical \& Electronics \\ Faculty of Engineering \\ Universiti Malaysia Sarawak \\ Kota Samarahan, Malaysia
}

\begin{abstract}
Phasor Measurement Units (PMUs) are normally used in Wide Area Measurement System (WAMS) for vast applications, such as monitoring the transmission system status over bulky areas including the control and protection. Therefore, this paper reviews the power system protection in relation to the synchronized PMUs and the applications of phasor estimation techniques. Furthermore, the paper elaborates the research works on the applications of PMU for diverse features. The structure and types of PMUs are described utilizing the mathematical modeling of simple Discrete Fourier Transform (DFT) with Recursive and NonRecursive estimation methods. The paper also presents the results of some published studies for two algorithms (Recursive and Non-Recursive). The review has revealed that the NonRecursive algorithm is more accurate and gives robust estimation results.
\end{abstract}

Keywords-Electrical Power System (EPS), “Global Positioning System (GPS),” PMU, Synchrophasors, Wide Area Measurement Protection and Control System (WAMPACS)

\section{INTRODUCTION}

Currently, EPS is advancing towards more complexity in which the transmission systems are the most assailable systems of the modern EPS. For the protection of transmission systems, power utilities use primary and backup protections through different relays and Intelligent Electronic Devices (IEDs) based techniques. Whereas, wide area measurement systems (WAMS) have fundamentally established on the advanced data procurement technology of phasor assessment. These systems permit monitoring and control of transmission line network status over bulky areas in sight of spotting grid instabilities [1], [2]. Current, voltage and frequency measurements which are GPS time-stamped, are taken by PMUs at tapped positions in the power networks. This data is stored in Phasor Data Concentrators (PDC) every 100 milliseconds, as it makes the grid completely observable. The phasors measured by PMUs at the same instant provide a real-time status of the bus-bars where they are located. Thus, a real-time surveillance of the bus-bars in power transmission networks helps the grid to be secured from the blackouts. Consequently, it is being contemplated as an important component in the future smart grid for cybersecurity and fault fortification.

In regard to the phasor estimation techniques, there are many researches presented on the DFT phasor estimation as reviewed in Sector II. But, when it comes to addressing the size of the sample data window, achieving a direct relationship between data processing speed and accuracy is still the main concern of the current research works. Indeed, the higher the data window the better the accuracy of

This work has been supported by the Digital Sarawak CoE grant (UHSB/B-AM2018/090), Sarawak State Government (Malaysia). estimation. However, it takes a longer time to compute such phasors when implementing full cycle DFT. Likewise, the half cycle DFT determines the phasor in a shorter time as it has a smaller window of data with the availability of less data for phasor extraction. Hence, a decrease in the accuracy of phasor computation can be observed. Due to the continuous time nature of the system, in the recently proposed Non-Recursive and Recursive DFT phasor techniques, the error occurrence in recursive computation tends to increase as recursive estimation computes each new sample from its old sample. Whereas the non-recursive estimation computes each new sample afresh.

The key purpose of this review paper is to analyse the studies pursued by different researchers and deliver vast details on the applications of PMU for EPS. This paper is organized as follows. A comprehensive review of the PMU applications including the phasor estimation and disturbance detection is presented in Section II. In Section III, the structure and PMU types are explained with a discussion on the traditional state estimation and PMU measurements. The recursive with non-recursive DFT algorithm is elaborated in Section IV. The performance of each method is discussed in Section V, and the conclusion is drawn in Section VI.

\section{LITERATURE REVIEW}

A.G. Phadke et al., [1] in 1977 developed the symmetrical component distance relay (SCDR) for the fortification of transmission lines and foreshadowed as the basis for the creation of the PMU. He mentioned that the equations derived uses the symmetrical components of line parameters, and is valid for all types of faults. In 1994, Wilson [3] demonstrated an analysis of the power flow through PMU on a $500-\mathrm{kV}$ transmission line in Northern California. Two PMUs were placed on both sides of the line for recording the voltage and current measurements. After comparing the angles found by the traditional formulation with the resulting angles of the PMU, a very satisfactory validation of results was obtained. Similarly, Wilson and Hauer [4], [5] demonstrated the effectiveness of PMU phasor data extraction, as an important step towards protecting transmission lines utilizing the differential philosophy.

Using the features of PMU Ma, Zhang, et al., in 2010 [6] led their research on tracing the source of disruption for the low-frequency oscillations. It was found that the forced low oscillations were either caused by the negative damping or resonance effect. With the applications of Power System Stabilizer (tuning or the intertie line controls), the negative damping effect can be reduced but, the resonance cannot be quenched with these techniques, as these types of oscillations alter rapidly. The survey writings about the applications of PMUs in the power grid shrouded the research and 